\title{
Study of the 27-day variations in the GCR fluxes in 2007-2008 based on PAMELA and ARINA observations
}

\section{R. Modzelewska ${ }^{1}$}

Siedlce University, Konarski Str.208110 Siedlce, Poland, e-mail: renatam@uph.edu.pl

\section{G.A. Bazilevskaya}

Lebedev Physical Institute, RAS, RU-119991Moscow, Russia,e-mail: gbaz@rambler.ru

\section{Boezio}

INFN, Sezione di Trieste, I-34149 Trieste, Italy, e-mail: boezio@trieste.infn.it

\section{S.V. Koldashov}

National Research Nuclear University MEPhI, RU-115409 Moscow, Russia, e-mail: svkoldashov@mephi.ru

\section{M.B. Krainev}

Lebedev Physical Institute, RAS, RU-119991 Moscow,Russia, e-mail: mkrainev46@mail.ru

\section{A.G. Mayorov}

National Research Nuclear University MEPhI, RU-115409 Moscow, Russia, e-mail: agmayorov@mephi.ru

\section{M.A. Mayorova}

National Research Nuclear University MEPhI, RU-115409 Moscow, Russia, e-mail: mamayorova@mephi.ru

\section{R. Munini}

INFN, Sezione di Trieste, I-34149 Trieste, Italy, e-mail: riccardo.munini@ts.infn.it

\section{I.K. Troitskaya}

National Research Nuclear University MEPhI, RU-115409 Moscow, Russia, e-mail: kote.electric@gmail.com

\section{R.F. Yulbarisov}

National Research Nuclear University MEPhI, RU-115409 Moscow, Russia, e-mail: rfyulbarisov@gmail.com

\section{On behalf of PAMELA collaboration}

36th International Cosmic Ray Conference -ICRC2019-

July 24th - August 1st, 2019

Madison, WI, U.S.A.

\section{${ }^{1}$ Speaker}




\begin{abstract}
We have investigated the energy and time dependences of the amplitude of the proton galactic cosmic ray intensity 27-days oscillations in 2007-2008 from the data of the PAMELA and ARINA spectrometers on board the RESURS DK-1 satellite. We have used different types of harmonic analysis, such as Fourier and wavelet analysis. The time profiles and amplitude dependences for the rigidities of cosmic ray particles from $\sim 300 \mathrm{MV}$ to tens of GV were obtained. It is shown that the amplitude-rigidity dependence cannot be described by a power law at low energies, the amplitude decreases with decreasing rigidity starting from $1 \mathrm{GV}$. For a physical interpretation, we have studied the relationship between the 27-day variations of GCR and heliospheric parameters: solar wind velocity and heliospheric magnetic field.
\end{abstract}

\title{
1. Introduction
}

The recurrent variations of the galactic cosmic ray (GCR) intensity and anisotropy which are due to the passage through the point of measurement of the solar wind (SW)-heliospheric magnetic field (HMF) structures rotating with the Sun, have been studied for more than 60 years [1]. According to the modern concept, the source of these structures is the longitudinal gradient of the SW velocity in the base of heliosphere, connected in its turn with the geometry of the fluxtube from the coronal holes to the heliosphere $[2,3]$. As a result in the inner heliosphere $(r<1 \mathrm{AU})$ the stream interaction regions (SIRs) are formed between the low-velocity stream around the heliospheric current sheet (HCS) and the overtaking it fast-velocity stream. At the greater distances $(r \approx 3-6 \mathrm{AU})$ this structure of the compressed HMF is expanding with two shock-waves around it forming the corotating interaction region (CIR) [4]. In case of stable position and power of the SIR's source near the Sun for 5-10 solar rotations (the synodic solar period $P_{\odot} \approx 27$ days), a CIR spreads over several AUs, and a 27-day variation arises in the GCR characteristics, in fact, occupying the region of the azimuthally inhomogeneous GCR intensity. Such situation is characteristic for the periods near the solar cycle minima.

In spite of the long history of observations, some characteristics of the 27-day variations are still not enough known [1,4], in particular, their dependence on the particle's rigidity in the wide energy range.

Although a genetic connection between the 27-day variation in GCR and CIRs is obvious, the mechanism of the 27-day GCR modulation is not quite clear because of multiplicity of processes involved. The timings of the CIR structures and the GCR flux modulation are closely connected. However, no parameter was found to be the only one or decisive factor for GCR modulation [5]. The 27-day GCR variation is still not well reproduced by the theoretical models [e.g., 6, 7]. Actually, a relative role of various mechanisms of modulation is now the most interesting point in the understanding of the 27 -day GCR variation.

This paper is devoted to the well-known episode of the 27-day GCR variation in 20072008 , near the minimum of solar cycle 23 , the period exclusively favorable for the development of the pronounced and long-lived recurrent GCR variation. This episode was extensively studied based on the observations with the neutron monitors (NMs) [e.g., 8-10] and the space probes [e.g., 11-13]. All authors stressed very stable period $\sim 27$ days and a strong negative correlation of the 27-day waves in GCR and solar wind velocity. Correlations with HMF strength and its components were less prominent.

The sequence of papers $[14,15,8]$ studied theoretically and successfully reproduced the 27-day variation of GCR observed by NMs during 2007-2008. Guo and Florinski [6, 7], Wiengarten et al., [16], and Kopp et al., [17] used MHD modeling of the SW and HMF serving 
as input to a GCR transport code employing a stochastic differential equation approach. In spite of undoubted progress, these models have some inconsistency with observations.

Here, we present the results of the 27-day variation in the GCR protons with rigidities from $\sim 0.3 \mathrm{GV}$ to $\sim 23 \mathrm{GV}$ as observed by the space-borne instruments PAMELA and ARINA in 2007-2008. We study the rigidity dependence of the amplitude of the 27-day GCR variation (A27) and discuss the results in the frame of GCR modulation mechanisms. The structure of the paper is as follows: in Section 2 the experiments PAMELA and ARINA are briefly described. The daily GCR intensities and their time behavior are discussed and the period of intense 27-day GCR variation is isolated in Section 3. Section 4 is devoted to the overall description of the situation with the 27-day variation in the heliospheric characteristics and GCR intensity in this period. In Section 5 is presented the method of processing and the preliminary results on the rigidity dependence of the amplitude of the 27-day variation in the proton intensities. The discussion is in Section 6.

\section{PAMELA and ARINA experiments}

The spectrometers PAMELA [18] and ARINA [19] situated on the same spacecraft Resurs DK1, operated during almost 10 years from June 2006. In 2007-2008, the satellite orbit was elliptical (altitude varying between 355 and $584 \mathrm{~km}$ ) with inclination of about $70^{\circ}$ and a period of about 94 minutes. The PAMELA instrument allowed a precise measurement of protons, electrons, their antiparticles, and light nuclei-in the kinetic energy interval from several tens of $\mathrm{MeV}$ up to several hundreds of $\mathrm{GeV}$. The instrument consisted of a magnetic spectrometer with a silicon tracking system, a time-of-flight system shielded by an anticoincidence system, an electromagnetic calorimeter and a neutron detector. The data treatment is described in rather detail by Munini et al., [20].

The ARINA telescope was a multilayer scintillation detector consisting of 10 plates arranged as a truncated pyramid. Particles were identified by the energy loss in each detector and the path until stopping measured in the number of plates ( $\mathrm{dE} / \mathrm{dX}$ vs $\mathrm{E}$ method). The instrument detected electrons with energies of 3-30 MeV and protons with energies of 30-110 MeV. The energy resolution of the ARINA spectrometer was 10-15\%. The aperture of the device was $\sim 10$ $\mathrm{cm}^{2}$ sr. For the extraction of the galactic component, events with energy higher than the geomagnetic cutoff were selected at each registration point, on L-shells no less than 8.

Here we concentrate on the investigation of the periodic variation of proton fluxes measured by PAMELA and ARINA in 2007-2008 taking place during prolonged solar minimum between solar cycles 23 and 24. We compare the PAMELA and ARINA observations with NMs data.

\section{Data treatment}

To study the 27-day GCR variation, daily proton fluxes of PAMELA and ARINA were normalized as $\frac{\left(x-\tilde{x}_{29 d}\right)}{\tilde{x}_{29 d}} \cdot 100 \%$ and de-trended (excluding moving average 29 days trend). Relative proton fluxes are presented in Fig. 1 for PAMELA (Fig.1a), ARINA (Fig.1b) and Oulu NM (Fig. 1c), respectively. Using the method adopted in [8] we calculated the power of the 27day variation for Oulu NM (Fig. 1d).

To study dynamics of the temporal changes of the periodicity connected with the Sun's 
rotation the wavelet time-frequency spectrum technique was used. In our calculation we adopted Morlet wavelet mother function [21]. In Figure 2 and 3 are presented results of wavelet analysis of the PAMELA proton flux for rigidity $1.8 \mathrm{GV}$ (Figure 2) and ARINA proton flux for rigidity $0.43 \mathrm{GV}$ (Figure 3).

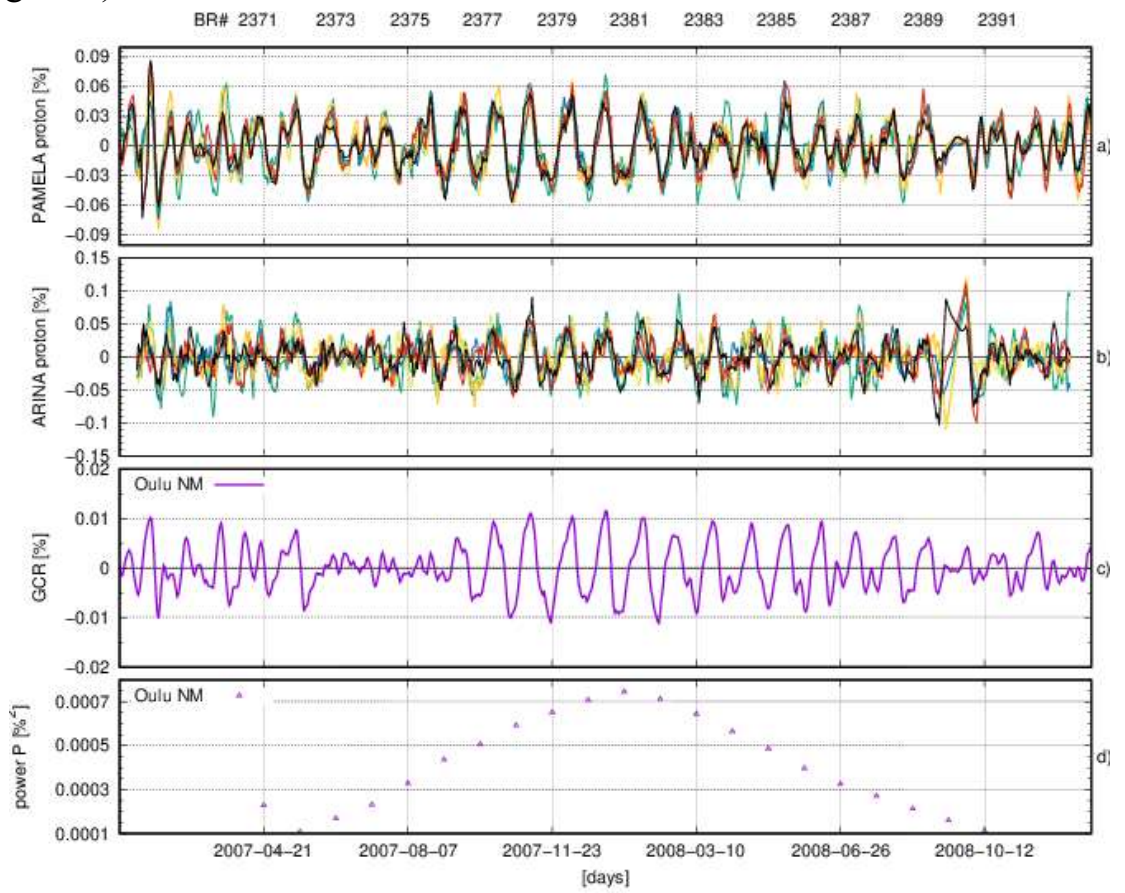

Figure 1: Temporal evaluation of the relative proton fluxes measured by PAMELA(a), ARINA(b) and Oulu NM (c); power P of the recognized periodicity 27-days for Oulu NM (d) in 2007-2008.

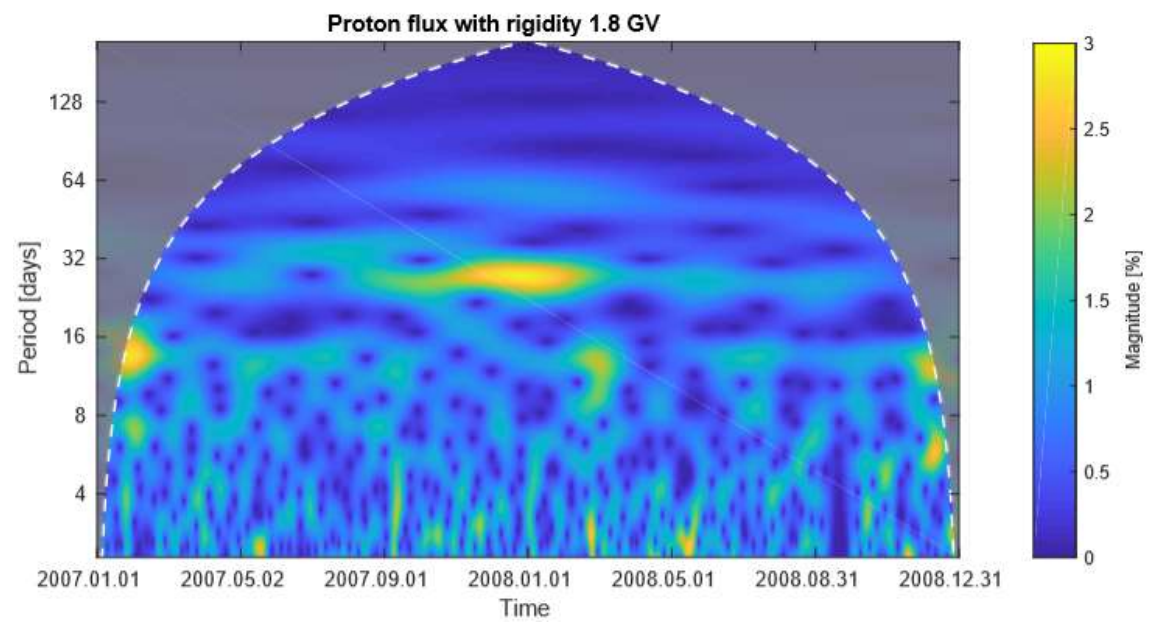

Figure 2: Results of wavelet analysis of the proton flux for rigidity 1.8 GV measured by PAMELA for 20072008. 


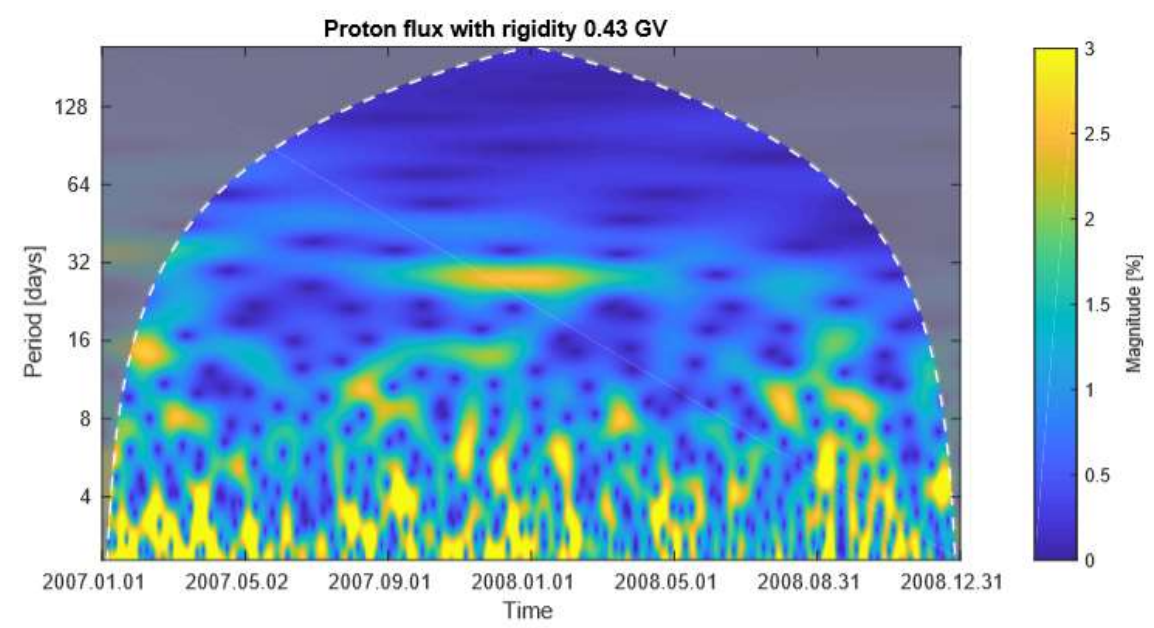

Figure 3: Results of wavelet analysis of the proton flux for rigidity $0.43 \mathrm{GV}$ measured by ARINA for 20072008.

Presented results confirm the high power and large amplitude of the 27-day GCR variation in the period of 30 September 2007 - 11 February 2008 corresponding to the Bartel rotations (BRs) 2377-2381. We choose this time interval for further analysis.

\section{Heliospheric and GCR characteristics in the period of intense 27-day variations in 2007-} 2008

In Figure 4 the time profiles of the relative de-trended characteristics of SW and HMF are compared with those of proton intensity according to PAMELA and Oulu NM .

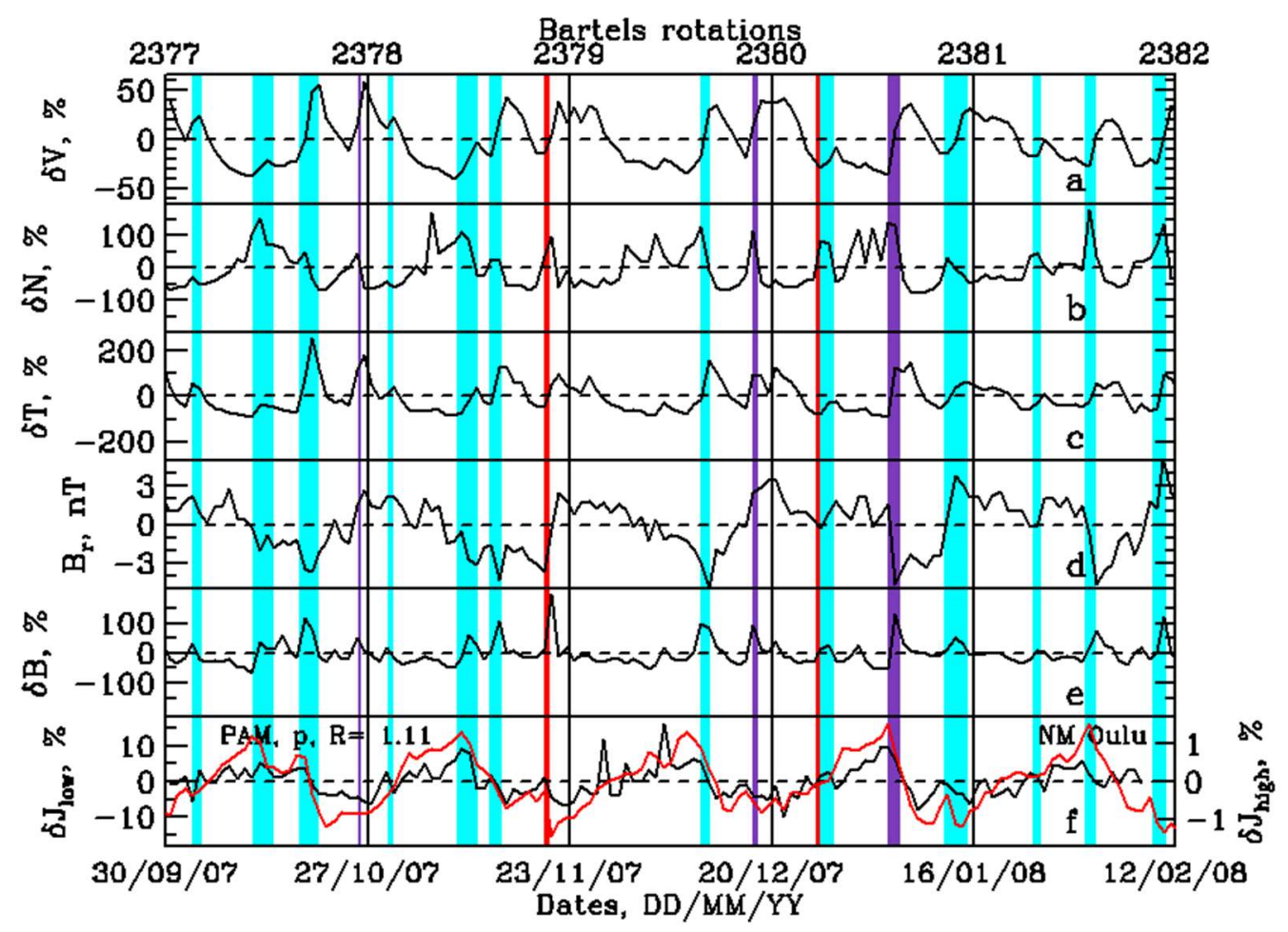


Figure 4: The SW and HMF characteristics and GCR intensity in the selected period. The relative de-trended characteristics are shown for all characteristics: SW (a - velocity, b - density, c - temperature), HMF (e strength) [OMNI], the radial HMF component (d) for which $B_{r}$ itself is shown. As the GCR intensities (f) those of maximum amplitude of the 27-day wave $(R \approx 1.1 \mathrm{GV}$, PAMELA, black, left y-axis) and according to Oulu NM (red, right y-axis) are shown. The SIRs (blue), CIRs (violet) and interplanetary coronal mass ejecta (ICME) (red) according to [http://www-ssc.igpp.ucla.edu/ jlan/ACE/Level3/] are shown as vertical bands.

A few facts can be inferred from Fig. 4. First, the period is rather quiet (only two weak ICMEs are observed in 137 days). Second, there are two distinct peaks in the SW velocity divided by two valleys of quite different duration: short valley in the end of each BR, correlating with the fast crossing of the low-velocity layer surrounding the HCS, and the long valley in the velocity in the middle part of each BR associated with the slow crossing of the HCS layer only slightly tilted to the Earth's trajectory. Third, there are SIRs in almost all cases of strong $\partial V / \partial t>0$ with corresponding peaks in the SW density, temperature and HMF strength. However, only 3 out of 15 interacting regions are accompanied by the shock waves, so they are CIRs near the Earth by our definitions. Fourth, the relative detrended GCR intensity both for rather low and high rigidities demonstrates more simple distribution than that of SW velocity: one broad minimum in the GCR intensity formed around two close velocity peaks and a broad maximum around long valley in the velocity. The amplitude of this GCR variation is quite different $(5 \%$ and $1 \%$, for low and high rigidities, respectively).

So the time behavior of the SW, HMF and GCR intensity near the Earth in the selected period of the intense 27-day variations can be considered as the manifestation of the stationary but longitudinally dependent variations in the coordinate system rotating with the Sun.

\section{Rigidity dependence of the 27-day GCR variation}

We have studied the rigidity dependence of the amplitude of the 27-day GCR variation in the interval of $0.3-20 \mathrm{GV}$ (A27) for proton flux measured by PAMELA and ARINA instruments for 5 BRs 2377-2381 corresponding to 30 September 2007 - 11 February 2008. The A27 were calculated by means of the normalized and de-trended daily data as the first harmonic of the Fourier extension using harmonic and wavelet methods. Errors are calculated as the standard deviation from the average value. In scope of assumed calculation accuracy, harmonic and wavelet methods give almost the same results within the accuracy of the procedures.

At the rigidities above $\sim 2 \mathrm{GV}$ a power-law dependence with an index $-0.72 \pm 0.49$ was found. In the interval 0.6-1.4 GV A27 demonstrated almost no $R$-dependence with a clear decrease at $R$ $<0.6 \mathrm{GV}$. This was corroborated by the ARINA observations which showed a minimum A27 at $0.4 \mathrm{GV}$. The preliminary results will be presented at the ICRC 2019.

Leske et al., [12], studied the recurrent variations of GCR and anomalous cosmic rays (ACR) measurements in the range from 10 to several hundred $\mathrm{MeV} / \mathrm{n}$. They found the mixing of highamplitude ACRs with low-amplitude GCRs. It seems to be a reason why they did not see a minimum in the rigidity spectrum below $1 \mathrm{GV}$. 


\section{Discussion}

The rigidity dependence of A27 was predominantly investigated on the base of NMs, i.e. at $R$ above several GV, where a power-law behavior was found with an index from 0.4 to 1.4 [9] which is consistent with our results. A clear maximum around $R \sim 1 \mathrm{GV}$ was initially observed in 19921994 during the out-of-ecliptic journey of the Ulysses spacecraft [22]. That time, the spatial and rigidity dependences of the recurrent GCR modulation and the latitudinal GCR gradient showed remarkable similarity [23] and it seemed that there must be a common modulation process controlling the flux variation in these phenomena. The adiabatic cooling effect by expanding solar wind was suggested to be such a mechanism [24]. GCR particles propagating from polar regions to the equatorial $(\mathrm{A}>0)$ continuously undergo adiabatic energy loss that results in the latitudinal gradient. However, the period of 2007-2008 belongs to the $\mathrm{A}<0$ epoch when the main proton propagation occurs in the heliospheric current sheet. The relationship between the 27-day GCR variation and the latitudinal gradient was not corroborated by the later observations [25]. Moreover, De Simone et al., [26], based on simultaneous observations by the PAMELA and Ulysses in 2006-2008, found a latitudinal gradient much smaller than predicted by current particle propagation models. The physical mechanism of the rigidity dependence of A27 as obtained from the PAMELA and ARINA observation remains to be under discussion.

\section{Acknowledgments}

We acknowledge partial financial support from The Italian Space Agency (ASI) under the program "Programma PAMELA - attivita' scientifica di analisi dati in fase E". We also acknowledge support from Deutsches fur Luft- und Raumfahrt (DLR), The Swedish National Space Board, The Swedish Research Council, The Russian Space Agency (Roscosmos), Russian Science Foundation and NASA Supporting Research Grant 13SRHSPH1320075 and the RFBR grants: 17-02-00584a, 18-02-00582a, 19-52-60003 SA-t. Neutron monitor count rates are from Neutron Monitor Data Base: http:/www01.nmdb.eu/. Data of heliospheric parameters are from http://omniweb.gsfc.nasa.gov and http://www-ssc.igpp.ucla.edu/ jlan/ACE/Level3/. R.M. acknowledges The Polish National Science Centre, decision number 2017/01/X/ST9/01023.

\section{References}

[1] J.A. Simpson, Space Sci. Rev. 83 (1998) 169-176

[2] Y.-M. Wang and Sheeley, N. R., Jr., ApJ. 355 (1990) 726

[3] C. N., Arge, and Pizzo, V. J., JGR 105 (2000) 10465

[4] J.G. Richardson, Living Rev. Sol. Phys. 15 (2018) 1

[5] A. Kumar, Badruddin, Solar Phys. 289 (2014) 4267-4296

[6] X. Guo and Florinsky V., Astrophys J 826 (2016) 13 pp

[7] X. Guo and Florinsky V., JGR 119 (2014) 2411-2429

[8] R. Modzelewska and Alania M V., Solar Physics 286 (2013) 593-607

[9] A. Gil and Alania M. V., Solar Physics 291 (2016) 1877-1886 
[10] A. Gil and Mursula K., J. Geophys Res. 123 (2018) 6148-6160

[11] J. Gieseler, N. Dresing, P. Dunzlaff, R. at al., Int. Cosmic Ray Conf. 1 (2009)

[12] R. Leske et al. Int. Cosmic Ray Conf. 11 (2011) 194

[13] M. Krainev et al., Physics of Atomic Nuclei, 81(9) (2018) 1355-1361

[14] M. V. Alania, R. Modzelewska, A. Wawrzynczak, Adv. Sp. Res 45 (2010) 421-428

[15] M. V. Alania, R. Modzelewska, A. Wawrzynczak, Solar Physics, 270 (2011) 629-641

[16] T. Wiengarten, Kleimann, J., Fichtner, H., et al., ApJ. 788 (2014) 80

[17] A.Kopp, Wiengarten, T., Fichtner, H. at al., Astrophysical J. 837 (2017) 37

[18] O. Adriani, et al., Phys. Reports 544(4) (2014) 323-370

[19] A.V., Bakaldin, Batishchev A.G., Voronov S.A., et al., Cosmic Research 455 (2007) 471

[20] R. Munini et al., 35th International Cosmic Ray Conference PoS(ICRC2017) 091

[21] C.Torrence and G. Compo, Bulletin of the American Meteorological Society, 79 (1998) 61-78

[22] R. B., McKibben, Simpson, J. A., Zhang, M., Bame, S., Balogh, A., Sp. Sci. Rev. 72 (1995) 403-408

[23] C. Paizis, Heber, B., Ferrando, et al., J. Geophys. Res. 104 (1999) 28241-28247

[24] M. Zhang, Astrophys. J. 488 (1997) 841-853

[25] P. Dunzlaff, Heber, B., Kopp, A., Rother, O., Mueller-Mellin, R., Klassen, A., et al., Ann. Geophys. 26 (2008) 3127-3138

[26] N. De Simone, Di Felice, V., Gieseler, J., Boezio, M., Casolino, M., Picozza, P., PAMELA Collaboration, and Heber, B., Astrophys. Space Sci. Trans. 7 (2011) 425-434 